Original research article

\title{
Three-layer collagen-based vascular graft designed for low- flow peripheral vascular reconstructions
}

\author{
Miroslav Špaček ${ }^{1 *}$, Hynek Chlup ${ }^{2}$, Petr Mitáš ${ }^{1}$, Jan Veselý ${ }^{2}$, Lukas Lambert ${ }^{3}$, Mikuláš Mlček ${ }^{4}$, \\ Milan Krajíček ${ }^{1}$, Jaroslav Lindner ${ }^{1}$, Tomáš Grus ${ }^{1}$ \\ ${ }^{1}$ Charles University and General University Hospital in Prague, First Faculty of Medicine, 2nd Department of Surgery - Department of Cardiovascular \\ Surgery, Prague, Czech Republic \\ ${ }^{2}$ Czech Technical University in Prague, Faculty of Mechanical Engineering, Department of Mechanics, Biomechanics and Mechatronics, Prague, \\ Czech Republic \\ ${ }^{3}$ Charles University and General University Hospital in Prague, First Faculty of Medicine, Department of Radiology, Prague, Czech Republic \\ ${ }^{4}$ Charles University, First Faculty of Medicine, Institute of Physiology, Prague, Czech Republic
}

\begin{abstract}
Introduction: The aim of this study was to develop a prototype of an artificial blood vessel which has similar mechanical properties to a human saphenous vein graft and to experimentally verify the function of the prosthesis via ovine carotid bypass implantation.

Material and methods: The prototype of an artificial graft prosthesis for low flow was developed and manufactured from a collagenous matrix and reinforcing polyester mesh. We compared the results of both the pressurisation and the mechanical stress evaluation tests of VSM with four types of hybrid vascular graft. The most similar graft (type II) was chosen for the first ovine model implantation.

Results: Dominant behavior e.g. mechanical response of VSM graft in plots of circumferential and axial stress during loading is observed in circumferential direction. Average results of used VSM showed area of ideal mechanical response and the properties of artificial blood vessels were fitted into this area. Developed graft remained patent after 161 days of follow up in ovine model.

Conclusions: The mechanical properties of the graft were designed and adjusted to be similar to the behaviour of human saphenous veins. This approach showed promising results and enhanced the final performance of the prosthesis.
\end{abstract}

Keywords: Collagen-based vascular graft; Low-flow peripheral vascular reconstruction; Artificial blood vessel; Human saphenous vein; Inflation-extension test

\section{Introduction}

One of the foundations of vascular reconstructive surgery is the blood vessel graft. Arterial allotransplants were first used for aneurysm surgery on the abdominal aorta. After a short period it was observed that, because of immunologically driven degenerative changes, the grafts became closed or a rupture occurred. In the early fifties (Kunlin, 1953) the use of autotransplant from saphenous vein (VSM) was favoured. Since then, the saphenous vein is most often used as an arterial bypass graft in coronary artery bypass surgery and, of course, in cases of below-knee arterial reconstruction. However, the use of VSM is for some patients limited by its dimensions, availability or pathology. An artificial graft may be a solution for these patients. The first prosthesis was manufactured in 1954 from synthetic fibres using textile procedures (Blakemore and Voorhees, 1954). Since that time, dynamic development has been observed in this area. Currently, artificial grafts manu- factured from different materials such as PTFE (expanded polytetrafluorethylene), Dacron etc., are available to be used in different anatomical areas of the human body (Ambler and Twine, 2018; Wang et al., 2007).

One of the biggest disadvantages of a prosthesis is its low patency when it is used for low flow reconstructions (Kannan et al., 2005). These limitations could be found in cases of flow lower than $100-200 \mathrm{ml}$ per minute. The second drawback is the sensitivity of polyester fibre towards infection.

Experience shows that their mechanical properties are crucial characteristics (Horný et al., 2014) of vein autotransplants used for low flow bypass. They are capable of creating a perfect anastomosis and also allow the graft to become adapted to the pressure and flow conditions in the arterial system. Therefore, the aim of artificial blood vessel development was to mimic the mechanical behaviour of saphenous vein grafts (Veselý et al., 2015a). The human saphenous veins were tested in order to determine the ideal range of mechanical properties which the prosthesis characteristics should fit. The properties of artificial

\footnotetext{
* Author for correspondence: Miroslav Špaček, Charles University and General University Hospital in Prague, First Faculty of Medicine, 2nd Department of Surgery - Department of Cardiovascular Surgery, U Nemocnice 2, 12808 Prague 2, Czech Republic; e-mail: mirekspacek@seznam.cz; http://doi.org/10.32725/jab.2019.002

Submitted: 2018-04-13 • Accepted: 2019-01-16 • Prepublished online: 2019-01-23

J Appl Biomed 17/1: 47-52 • EISSN 1214-0287 • ISSN 1214-021X

(c) 2019 The Authors. Published by University of South Bohemia in České Budějovice, Faculty of Health and Social Sciences.

This is an open access article under the CC BY-NC-ND license.
} 
graft were then tested and tuned, including the influence of sterilisation process.

The aim of this study was to develop a prototype of an artificial blood vessel which has mechanical properties similar to saphenous veins grafts (Singh et al., 2015) and experimentally verify the function of the prosthesis using implantation into a sheep model. The authors aimed to create an ideal bypass graft for arterial bypass surgery, best resembling the mechanical properties to a human venous graft, which to date is the best graft available in human reconstructive vascular surgery. Arterial grafts used in cardiac surgery with excellent results are limited by their length (up to 15-20 cm), and they are not sufficient for use in infrainguinal bypass surgery in patients presenting with critical limb ischaemia.

\section{Material and methods}

\section{Human saphenous veins}

Samples of human great saphenous vein (SV) were collected either during coronary-artery bypass surgery conducted at the General University Hospital in Prague (obtained with informed consent) or during autopsies conducted at the Department of Forensic Medicine of the Third Faculty of Medicine of Charles University in Prague. The experimental protocol was approved by the institutional Ethical Committees. Eleven samples were tested within 72 hours after excision (mean \pm SD; age $62 \pm 8.2$; 9 male and 2 female; 8 samples from surgery and 3 from cadavers). The obtained mechanical response of the veins was used to determine a reference range within which the response of artificial prosthesis should fall (Veselý et al., 2015a).

\section{Matrix for artificial blood vessel}

Eleven different polyvinyl alcohol (PVA) preparations in total were tested. The preparations differed in molecular weight, level of hydrolysis, branching, etc. The PVA was then mixed in different ratios with denatured and native collagen type I (VUP Brno) and gelatin (G9382, gelatin from bovine skin, 225 g Bloom, Type B, Sigma-Aldrich). The first selection process was performed using tests of the mechanical properties, grade of homogeneity, and solubility. Finally, the blend of denatured collagen and PVA (average $\mathrm{Mw}$ 130,000, $99+\%$ hydrolysed, Sigma-Aldrich) showed the best properties and was chosen for the next steps of development. Glycerol was used as a plasticiser of the blends. Hydrogels of these blends can mimic the structure of native blood vessels and have a better mechanical strength than laboratory derived collagen. These samples are insoluble in water (because of their relatively high molecular weight) without additional cross-linking process. The mechanical properties of these specimens were evaluated using uniaxial tensile tests (Horný et al., 2014).

\section{Artificial blood vessel}

The developed artificial blood vessel is a hybrid composite, as this arrangement has already been proven as advantageous over others (de Valence et al., 2012). A mesh from monophilic polyester fibres was used as a scaffold (made in-house). The blend of denaturated collagen and PVA was used as a matrix. The scaffold mesh was integrated into the matrix using extrusion process. The procedure allows for the creation of a prosthesis with different sandwich arrangement (different position of the scaffold in the matrix, different thickness of matrix). The created tubular samples were dried and sterilised by gamma irradiation using standard medical procedures. All of the manufactured prostheses were sterilised by $28 \mathrm{kGy}$ of gamma radiation.

The mechanical properties of the prosthesis were given by its internal composition. Four different arrangements of artificial blood vessel were tested (Fig. 1). Type I was manufactured from the collagenous matrix only (without the reinforcement of polyester mesh). The polyester mesh was placed on the outer diameter of the specimen for the type II. Type III and IV had the mesh integrated inside the collagenous matrix. For the type III the outer layer was thinner than the inner one (ratio of $40: 60$ percent). Type IV was arranged in the opposite way, with the inner layer being thinner than the outer one.

\section{Pressurisation tests}

Both the human saphenous vein and the artificial blood vessel samples were tested using the inflation-extension test. The mechanical response of the prosthesis was adjusted to mimic the behaviour of native veins. This test was chosen because the filling of the specimen is similar to physiological filling of blood vessels.

Each specimen was marked with a black liquid eye-liner, cannulated at one end, hung vertically in the experimental setup (Fig. 2) and pressurised by a motorised syringe up to approx. $20 \mathrm{kPa}$ (150 $\mathrm{mmHg}$ ). The prostheses were expanded to a
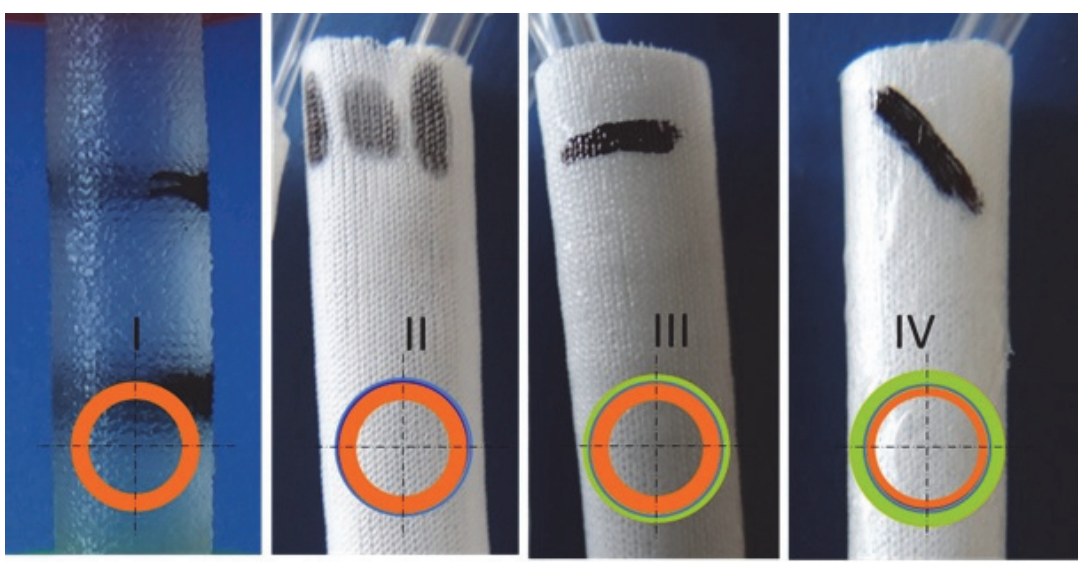

Fig. 1. Different types of manufactured and tested artificial blood vessel samples.

Blue - reinforcing polyester mesh; green - outer layer of collagenous matrix; orange - inner layer of collagenous matrix (for colour resolution see the on-line edition). 
pressure which was approx. 5 times higher than the veins in order to confirm and verify the safety of their construction. The speed of the syringe piston was $0.1 \mathrm{~mm} / \mathrm{s}$. The intraluminal pressure was monitored by a pressure transducer (Cressto s.r.o, Czech Republic). The deformed geometry was recorded by a CCD camera (Dantec Dynamics, Skovlunde, Denmark). In the data post-processing, changes in the length between the black marks (Fig. 1) and average changes in the silhouette between markers were determined by an edge detection algo- rithm in Matlab (MathWorks, MA, USA). The obtained data were used to compute deformations in the axial and circumferential direction. The experiments were performed at room temperature $\left(22{ }^{\circ} \mathrm{C}\right)$. Prior to mechanical testing, two rings were cut out of the sample at both ends, and the mean reference dimensions of the samples (external radius, thickness) were determined by means of image analysis of digital photographs (Nis-Elements, Nikon Instruments Inc., NY, USA).

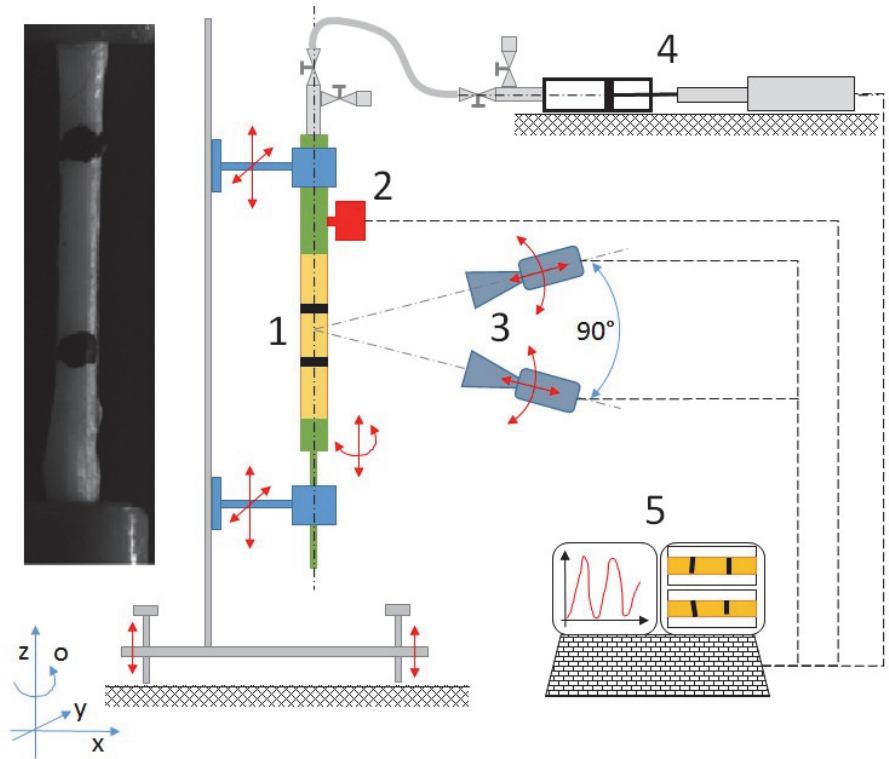

Fig. 2. Inflation test set-up: 1, sample; $\mathbf{2}$, pressure transducer; $\mathbf{3}$, CCD camera system; $\mathbf{4}$, pulsator, $\mathbf{5}$, measuring and controlling PC. Left: Photograph of the sample in front of contrasting background from CCD camera. Black marks were used to evaluate the longitudinal deformation of the sample.

\section{Mechanical stress evaluation}

The samples were considered to be incompressible cylindrical thin-walled tubes. The kinematics of the experiment was modelled as simultaneous inflation and extension. The longitudinal $\left(\lambda_{z}\right)$ and circumferential $\left(\lambda_{o}\right)$ stretch ratios were computed using the same equations as in previous papers (Veselý et al., 2014; 2015a, b). Using the incompressibility condition, relationship for stretch and conditions of equilibrium forces in the axial direction, the Laplace equation can be rewritten in form (1) and (2).

$$
\begin{gathered}
\sigma_{o}=\frac{r p}{h}=\frac{R \lambda_{o}{ }^{2} \lambda_{z} p}{H}=\frac{R\left(\varepsilon_{o}+1\right)^{2}\left(\varepsilon_{z}+1\right) p}{H} \\
p \pi R^{2}-\sigma_{z} 2 \pi R h=0 \sigma_{z}=\frac{\sigma_{o}}{2}
\end{gathered}
$$

In (1), $H$ is the reference thickness of the sample.

\section{Evaluation of prosthesis in the animal model}

The best mechanical performance was achieved by prosthesis type II. The prosthesis was implanted as an end-to-side graft (bypass) to ovine right carotid artery, which was ligated before bypassing. We administered standard perioperative drugs including Acetylsalitic acid, which was given for the first seven days following the operation (Anopyrin $100 \mathrm{mg}$, Zentiva, Czech Republic). The sample was explanted after 161 days and a macroscopic evaluation was performed.

\section{Results}

\section{Diameters of VSM and SCT}

The inner diameter of the tubes (SCT) was between 5.5$6.0 \mathrm{~mm}$. The approximate wall thickness of the tubes was $0.56 \mathrm{~mm}$ in VSM and $0.41 \mathrm{~mm}$ in SCT (Table 1 and Table 2).

Table 1. Diameters of the sandwich collagen tubes

\begin{tabular}{lccc}
\hline Sample & $R e_{S C T}(\mathrm{~mm})$ & $R_{S C T}(\mathrm{~mm})$ & $H_{S C T}(\mathrm{~mm})$ \\
\hline type I & 3.21 & 3.01 & 0.40 \\
type II-1 & 3.24 & 3.03 & 0.43 \\
type II-2 & 3.27 & 3.10 & 0.34 \\
type III-1 & 3.49 & 3.30 & 0.39 \\
type III-2 & 3.50 & 3.34 & 0.31 \\
type IV-1 & 3.45 & 3.18 & 0.54 \\
type IV-2 & 3.46 & 3.23 & 0.45 \\
mean & $\mathbf{3 . 3 7}$ & $\mathbf{3 . 1 7}$ & $\mathbf{0 . 4 1}$ \\
SD & $\mathbf{0 . 1 3}$ & $\mathbf{0 . 1 3}$ & $\mathbf{0 . 0 8}$ \\
\hline
\end{tabular}

$S C T$ - sandwich collagen tube, $R e_{S C T}$ - external radius of SCT, $R_{S C T}$ mean radius of SCT, $H_{S C T}$ - thickness of SCT. 
Table 2. Diameters of the saphenous vein grafts samples

\begin{tabular}{lcccccc}
\hline Sample & $\begin{array}{c}\text { Age } \\
\text { (years) }\end{array}$ & Sex & $\begin{array}{c}R e_{V S M} \\
(\mathrm{~mm})\end{array}$ & $\begin{array}{c}R_{V S M} \\
(\mathrm{~mm})\end{array}$ & $\begin{array}{c}H_{V S M} \\
(\mathrm{~mm})\end{array}$ & $\begin{array}{c}\text { Sample } \\
\text { of }\end{array}$ \\
\hline VSM1 & 63 & $\mathrm{M}$ & 2.89 & 3.31 & 0.83 & operation \\
VSM2 & 69 & $\mathrm{M}$ & 1.14 & 1.44 & 0.59 & operation \\
VSM3 & 63 & $\mathrm{M}$ & 2.05 & 2.43 & 0.76 & operation \\
VSM4 & 60 & $\mathrm{M}$ & 1.80 & 2.07 & 0.63 & operation \\
VSM5 & 76 & $\mathrm{M}$ & 1.98 & 2.27 & 0.57 & operation \\
VSM6 & 50 & $\mathrm{M}$ & 2.33 & 2.62 & 0.57 & operation \\
VSM7 & 60 & $\mathrm{M}$ & 1.92 & 2.16 & 0.47 & operation \\
VSM8 & 49 & $\mathrm{~F}$ & 1.86 & 2.06 & 0.39 & operation \\
VSM9 & 72 & $\mathrm{~F}$ & 1.78 & 2.14 & 0.71 & cadaver \\
VSM10 & 60 & $\mathrm{M}$ & 0.92 & 1.06 & 0.28 & cadaver \\
VSM11 & 60 & $\mathrm{M}$ & 2.14 & 2.35 & 0.42 & cadaver \\
Mean & $\mathbf{6 2}$ & & $\mathbf{1 . 8 9}$ & $\mathbf{2 . 1 7}$ & $\mathbf{0 . 5 6}$ & \\
sD & $\mathbf{8}$ & & $\mathbf{0 . 5 3}$ & $\mathbf{0 . 5 8}$ & $\mathbf{0 . 1 7}$ & \\
\hline
\end{tabular}

$V S M$ - vena saphena magna, $R e_{V S M}$ - external radius of VSM, $R_{V S M}$ mean radius of VSM, $H_{V S M}$ - thickness of VSM.

\section{Pressurisation tests of saphenous veins}

The mechanical properties of human saphenous vein samples, curves stress-deformation, in the circumferential and axial directions are shown in Fig. 3 and Fig. 4, respectively.

It was observed that the deformation in circumferential direction is approx. 10 times higher than in the longitudinal direction. These findings lead to the assumptions that the dominant behaviour is observed in the circumferential direction. Therefore, in Fig. 3, the range of ideal mechanical response was created and the properties of artificial blood vessels were adjusted to fall within this range. In order to compare the mechanical response with the response of alternative vascular substitutes, the samples of VSM 1 and VSM 4 (healthy typical saphenous vein grafts) were used as representatives - Fig. 3. They were placed in the middle of all the tested examples and the age of donors was very close to the median of age of all tested donors, as well.

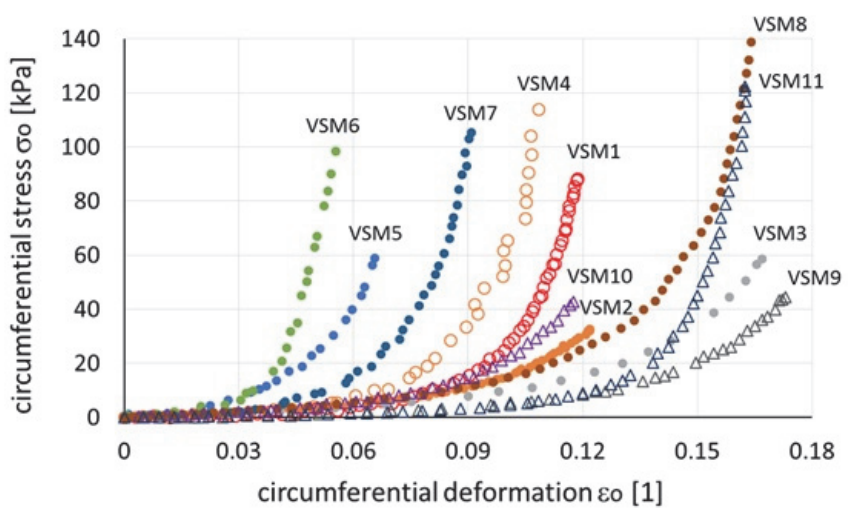

Fig. 3a. Stress-strain curve for human saphenous veins in the circumferential direction. The area of mechanical behavior into which the response of prosthesis was fitted is shown.

\section{Pressurisation tests of artificial blood vessels}

The mechanical response of the prosthesis samples is shown in Fig. 4 and Fig. 5. Type I is a simple collagenous tube with unacceptable deformation under low pressure. Type II shows a non-linear stress-deformation dependence which corresponds best to the reaction demonstrated by a vein. Type III and IV were more rigid, and this characteristic does not allow physiological pulse wave propagation.

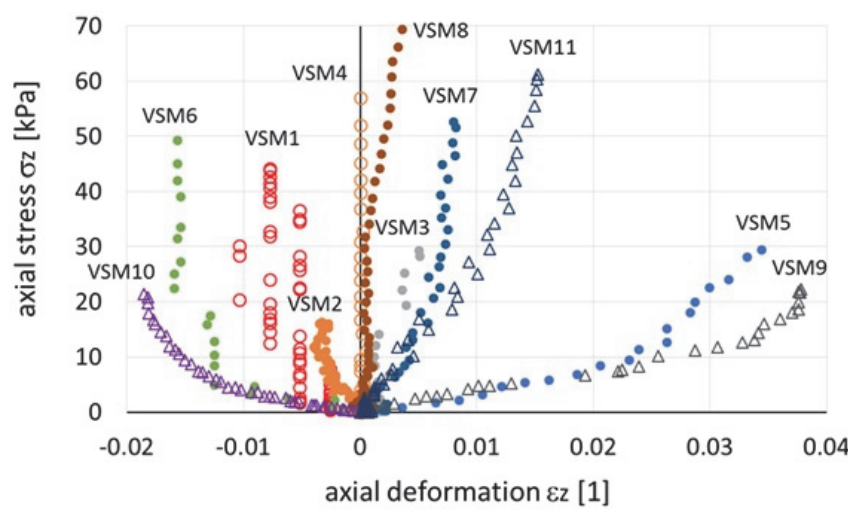

Fig. 3b. Stress-strain curve for human saphenous veins in the axial direction. VSM 1 and VSM 4 are representative characteristics of healthy v. saphena magna (they did not show any type of degeneration and were retrieved from healthy donors of mean age $62 \pm 8$ years). The area of mechanical behaviour is between characteristics of examples VSM 6 and VSM 3 (VSM 9). The nearest behaviour was seen in type II prosthesis.

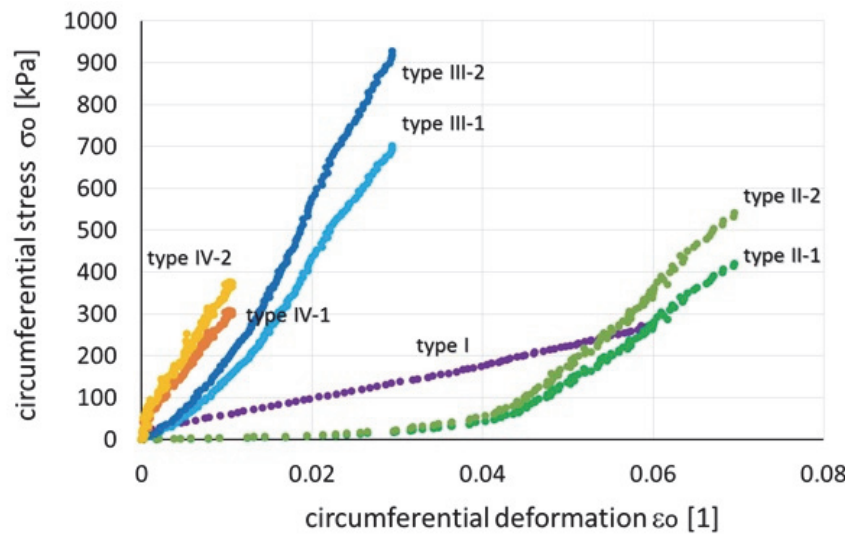

Fig. 4. Stress-strain curves in the circumferential direction for prosthesis with different internal arrangement

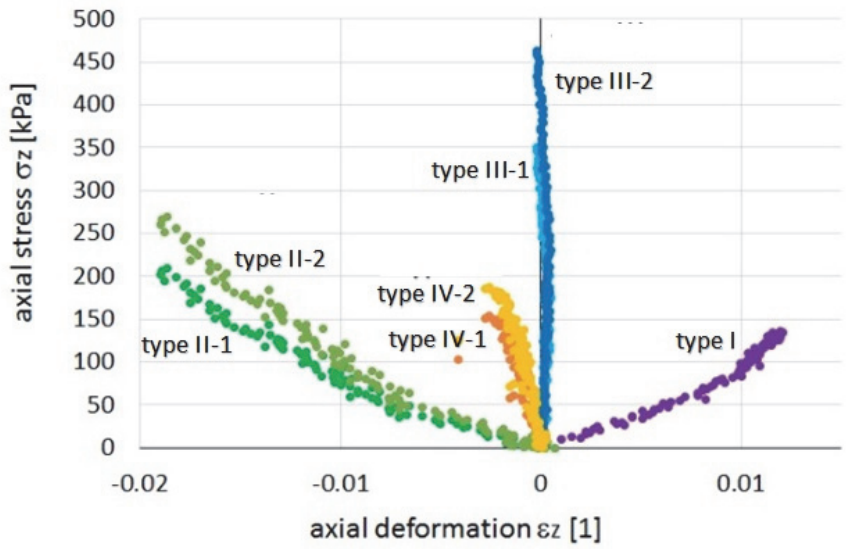

Fig. 5. Stress-strain curves in the axial direction for prosthesis with different internal arrangement 


\section{Model}

The first experimental implantation of the newly designed vascular prosthetic graft was carried out on an ovine model in the form of a carotid bypass (Fig. 6). The operation time was 3.4 hours, and the animal did not experience any perioperative complications or wound healing issues. The blood flow after sling placement was $145 \mathrm{ml} / \mathrm{min}$. Explantation of the patent graft was made on post-operative day 161 . The graft after removal was free of thrombi, with no signs of stenosis or any type of degeneration, including aneurysm formation.
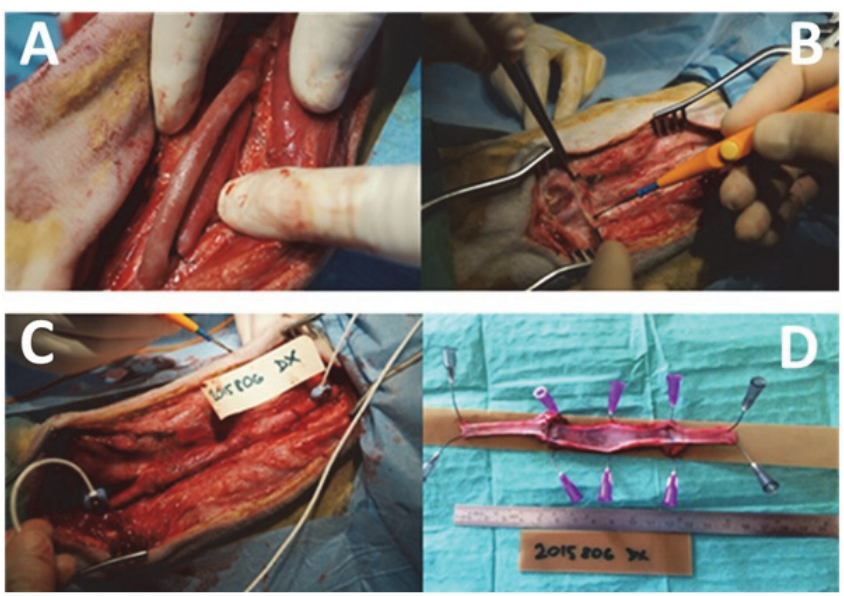

Fig. 6. The implantation of prosthesis into the ovine carotid artery and the condition after 161 days is shown. A, proximal and distal anastomosis End-to-Side, ligation of carotid artery; $\mathbf{B}$, explantation of patent graft; $\mathbf{C}$, flow measurement; D, no macroscopic signs of intimal hyperplasia after 161 days.

\section{Discussion}

Collagen is the most abundant biological material used for tissue engineering. It is the basic constituent of skin, bones, ligaments and connective tissues. Collagen-based biomaterials have been studied extensively for a variety of biomedical applications including dialysis membranes, wound dressings and artificial skin (Khan et al., 2011; Ravi and Chaikof, 2010; Sarkar et al., 2007). Although native collagen possesses a high tensile strength, the chemical treatment necessary for isolation makes the mechanical properties of reconstituted collagen very poor. A possible means to circumvent the problem is to reinforce the natural polymer matrix with a synthetic PVA polymer. The biggest advantages of PVAs are their mechanical performance, low price, and easy method of creating fibres. The non-antigenicity, non-toxicity, non-carcinogenicity, and non-allergy of this material has been repeatedly proven and therefore it is one of the main constituents used in the prosthesis manufacture process (Veselý et al., 2015b).

The high porosity of the inner layer reduces graft compliance due to fibrovascular infiltration of the wall, and the low porosity impedes the formation of the neointimal lining (Williams et al., 2011). The coating of the inner layer with an endothelial cell lining (endothelisation) generally improves the durability of prosthetic vascular grafts and can be further facilitated by modifying the epitopes of the surface and tissue engineering (Heyligers et al., 2005). During graft development, the thickness of the fibres and polyester mesh manufacturing were chosen in order to not influence the perfusion of the nutrients from the lumen to the outer layer of the graft (to avoid perfusion shadows). The unique extrusion process, performed on a special extrusion machine, allowed for the mesh integration into a homogenous matrix. The advantage of this process is, in terms of resistance to longitudinal dissection, a probably higher resistance to infection due to protection of the prosthetic scaffold from first time-contact with bacteria during implantation. The pressure wave distribution also seems to be more physiological in developer type II of the prosthesis. The theoretical advantages of our prosthesis over those already clinically used are mainly its compliance and the possibility for a more physiological distribution of the pressure wave. In our opinion, the graft exhibits a type of biointegration into the surrounding tissue, which is a major clinical sign of the absence of infection. This biointegration was clearly visible during the explantation surgery.

The prosthesis created from different layers could be programmed in its mechanical properties (Greenwald and Berry, 2000). Three processes are controlled in order to obtain the desired mechanical response. The first is the quality and mechanical performance of the collagenous matrix (the amount of collagen and PVA in the blend) (Veselý et al., 2015c). The second is the geometry of the polyester mesh (mesh size, fibre diameter, amount of fibres), and the third is the sandwich arrangement of the prosthesis layers. This study proved that the integration of the polyester mesh showed a significant influence on the final mechanical properties. Tests showed that the thicker the outer layer of prosthesis, the more compliant the whole structure is (Fig. 4). The arrangement of type II was, for the physiological filling, closest to the desired range of values set by the behaviour of veins (Fig. 4). Contrary to that, type IV showed very rigid behaviour. It should also be mentioned that the response of saphenous veins is highly nonlinear (Fig. 3). The same trend was again achieved by graft type II (Fig. 4).

To date there are three main drawbacks of currently used vascular prostheses - poor mechanical properties (compliance mismatch leading to intimal hyperplasia), susceptibility to infection, and poor hemocompatibility (thrombogenicity) resulting from the absence of an endothelial layer - the aim of this study is to address these mechanical properties.

The limitation of this study, however, is that it addresses only one of the parameters of vascular prostheses development and not the others. Other potential limitations include the fact that so far only the first, animal implantation was carried out and also the fact that the number of the performed measurements is relatively limited, although the dataset is rather unique. In our study we did not seed endothelial cells prior to implantation. Potential post-implant endothelialisation of our grafts is a topic for future research.

The effort to mimic the physiological behaviour of saphenous veins has shown to be a good strategy in the artificial blood vessel development process. Further testing in animal models and future clinical study, as well as necessary detailed histologic studies, will fully test the artificial graft proper function.

\section{Conclusions}

We designed an artificial blood vessel as a hybrid composite, where a mesh from monophilic polyester fibres was used as a scaffold and a blend of denaturated collagen and PVA was used as a matrix. The scaffold mesh was integrated into the matrix using an extrusion process. The mechanical properties of the prosthesis were tested in four different arrangements. The 
dominant behaviour of the human saphenous vein graft was in the circumferential direction.

We compared the results of the pressurisation and mechanical stress evaluation tests of the human saphenous vein graft with four types of hybrid vascular graft. The graft which best resembled the mechanical properties of human saphenous vein graft was chosen for the first in-vivo implantation, which was successful. The graft remained fully patent as a carotid bypass after 161 days of follow up in the ovine model and shoved no signs of degeneration after removal.

\section{Conflict of interests}

The authors have no conflict of interests to declare.

\section{Acknowledgements}

This work was supported by the Ministry of Health of the Czech Republic under AZV ČR 15-27941A.

These authors (Miroslav Špaček and Hynek Chlup) contributed equally to this work.

Dedicated to the memory of doc. MUDr. Milan Krajíček, DrSc.

\section{References}

Ambler GK, Twine CP (2018). Graft type for femoro-popliteal bypass surgery. Cochrane Database Syst Rev11 (2): CD001487. DOI: 10.1002/14651858.CD001487.pub3

Blakemore AH, Voorhees AB Jr. (1954). The use of tubes constructed from vinyon $\mathrm{N}$ cloth in bridging arterial defects; experimental and clinical. Ann Surg 140(3): 324-334.

de Valence S, Tille JC, Giliberto JP, Mrowczynski W, Gurny R, Walpoth BH, Möller M (2012). Advantages of bilayered vascular grafts for surgical applicability and tissue regeneration. Acta Biomater 8(11): 3914-3920. DOI: 10.1016/j.actbio.2012.06.035.

Greenwald SE, Berry CL (2000). Improving vascular grafts: the importance of mechanical and haemodynamic properties. J Pathol 190(3): 292-299. DOI: 10.1002/(SICI)10969896(200002)190:3<292:AID-PATH528>3.0.CO;2-S

Heyligers JM, Arts CH, Verhagen HJ, de Groot PG, Moll FL (2005). Improving small-diameter vascular grafts: From the application of an endothelial cell lining to the construction of atissue-engineered blood vessel. Ann Vasc Surg 19(3): 448-456. DOI: 10.1007/ s10016-005-0026-0.

Horný L, Netušil M, Voňavková T (2014). Axial prestretch and circumferential distensibility in biomechanics of abdominal aorta. Biomech Model Mechanobiol 13(4): 783-799. DOI: 10.1007/ s10237-013-0534-8.

Kannan RY, Salacinski HJ, Butler PE, Hamilton G, Seifalian AM (2005). Current status of prosthetic bypass grafts: A review. J Biomed Mater Res B Appl Biomater 74(1): 570-581. DOI: $10.1002 / j b m . b .30247$.

Khan R, Khan MH, Bey A (2011). Use of collagen as an implantable material in the reconstructive procedures - an overview. Biol Med 3(4): 25-32.

Kunlin J (1953). Venous grafts. J Int Chir 13(3): 313-319.

Ravi S, Chaikof EL (2010). Biomaterials for vascular tissue engineering. Regen Med 5(1): 107-120. DOI: 10.2217/rme.09.77.

Sarkar S, Schmitz-Rixen T, Hamilton G, Seifalian AM (2007). Achieving the ideal properties for vascular bypass grafts using a tissue engineered approach: a review. Med Biol Eng Comput 45(4): 327-336. DOI: 10.1007/s11517-007-0176-z.

Singh C, Wong CS, Wang X (2015). Medical textiles as vascular implants and their success to mimic natural arteries. J Funct Biomater 6(3): 500-525. DOI: 10.3390/jfb6030500.

Veselý J, Hadraba, D, Chlup H, Horný L, Adámek T, Žitný R (2014). Constitutive modelling and histology of vena saphena. Appl Mech Mater 486: 249-254.

Veselý J, Chlup H, Krajíček M, Žitný R (2015a). Mechanical properties of biological composite reinforced by polyester mesh. In: 53rd Experimental stress analysis. Praha: Czech Technical University in Prague, pp. 466-468.

Veselý J, Horný L, Chlup H, Adámek T, Krajíček M, Žitný R (2015b). Constitutive modeling of human saphenous veins at overloading pressures. J Mech Behav Biomed Mater 45: 101-108. DOI: 10.1016/j.jmbbm.2015.01.023.

Veselý J, Horný L, Chlup H, Beran M, Krajíček M, Žitný R (2015c). Effect of polyvinyl alcohol concentration on the mechanical properties of collagen/polyvinyl alcohol blends. Appl Mech Mater 732: 161-164. DOI: 10.4028/www.scientific.net/AMM.732.161.

Wang X, Lin P, Yao Q, Chen C (2007). Development of small-diameter vascular grafts. World J. Surg 31(4): 682-689. DOI: 10.1007/ s00268-006-0731-z.

Williams SK, Kleinert LB, Patula-Steinbrenner V (2011). Accelerated neovascularization and endothelialization of vascular grafts promoted by covalently bound laminin type 1 . J Biomed Mater Res A 99(1): 67-73. DOI: 10.1002/jbm.a.33138. 